ZUR

\title{
UIGURENFRAGE
}

vON

\section{W. SCHOTT.}

\section{AUS DEN ABHANDLUNGEN DER KÖNIGL. AKADEMIE DER WISSENSCHAFTEN ZU BERLIN 1875.}

ZWEITE ABTEILUNG.

\section{BERLIN.}

BUChDRUCKerei DeR KÖNigl. AKAdEMie der wissenschaften (G. vogt). UNIVERSTTÄTSSTRASSE 8 .

1875 .

IN COMMISSION BEI F. DÜMMLER'S VERLAGS-BUCHHANDLUNG (BARRWITZ UND GOSSMANN). 
Gelesen in der Akademie der Wissenschaften am 21. Januar 1875.

Die Seitenzahl bezeichnet die laufende Pagina des Jahrgangs 1875 in den Abhandlungen der philosophisch-historischen Klasse der Königl. Akademie der Wissenschaften. 